\title{
Another nail in the biosimilar coffin?
}

\author{
The US Food and Drug Administration (FDA) has raised the bar very high for those who seek to make a business out \\ of biosimilars.
}

ast month, the FDA released three draft guidance documents Ldetailing the scientific and quality issues that the agency regards as important in developing a biosimilar. Although they provide clarification on the depth of analytical characterization required for biosimilar approval, the guidances leave unclear what data are needed to prove interchangeability-one of the biggest incentives for biosimilar manufacturers.

Essentially, the new FDA process is a sequential two-step procedure. In the first, manufacturers seeking to market a biosimilar will have to demonstrate-largely with physicochemical and preclinical evidence-that they have a molecule that is 'biosimilar' to an onmarket reference compound (that is: "Does your erythropoietin look and smell like Epogen?").

The second step proposed in the draft guidance is to show just how similar-in clinical performance-a biosimilar is to the reference product. In other words, "If it acts like Epogen as well as looking and smelling like Epogen, then, by golly, it is biosimilar Epogen."

It is in these outline requirements for clinical similarity that the FDA ratchets up the bar. Stealing directly from the still constitutionally untested text of President Obama's Biologics Price Competition and Innovation Act (part of the Patient Protection and Affordable Healthcare Act), the FDA draft guidelines require a stringently defined standard of interchangeability - a designation that allows pharmacists to automatically substitute biosimilars for brands without the need for a physician's prescription.

Interchangeability could merely have meant that a sponsor of a biosimilarity clinical trial would have to show that its version of a compound has a spectrum of activity in a given population that is indistinguishable from what the reference product shows in that same population. This would have aligned the US proposals with the biosimilar guidelines currently operating in the European Union: the European Medicines Agency (EMA) recommends a two-arm clinical trial design (reference compound and trial agent) in a small number of similar people (clones would be ideal, if a little controversial for the EMA to have explicitly described). If the two arms of the trial produce a similar result, then a biosimilar can be approved.

The FDA guidelines go much further, however. The current text says that the biosimilar is expected to produce the same clinical result as the reference product "in any given patient" and that switching between therapies in that patient must be as seamless in terms of safety and efficacy as simply continuing with uninterrupted administration of the reference biologic.

At one level, this sounds like a reasonable and scientifically testable approach. The similarity of biosimilars would certainly be irrefutable if they performed just like the reference product. And it would be very feasible to design a suitably revealing crossover clinical trial to test interchangeability. By selecting patients representing a spectrum of response to the reference compounds, a broad range of patient types could be surveyed efficiently. Fifty or fewer patients might suffice for such a study, even to show similarity for a reference product that was used across many indications.

However, the way in which the FDA is currently stating its requirements in the draft looks less like a way of trying to define similarity and more like a call for identity with the reference product.

Furthermore, there is a practical difficulty in conducting crossover studies in which the patient cohort in each arm of the trial can be counted on the fingers of one finger. (To be clear, a 50-person trial in which each patient is switched (or not) between reference and test product is a trial with 50 arms.) Just how can such a trial compensate for the day-to-day variability in the interaction between patient and drug caused by diet, disease and other lifestyle factors?

Thus, 17 years after alternative versions of brand biopharmaceuticals were first discussed in this journal, the FDA has finally coughed up draft biosimilar guidances that move us closer to a complete formal pathway for biosimilar manufacturers.

Paradoxically, the companies that will welcome these documents with the greatest aplomb are biologics originators. They will find in the guidances a pleasingly detailed and potentially arduous path for any biosimilar manufacturer hoping to cut into a market share of their brand product. Such US brand companies may also see the clarifications about the FDA's biosimilar pathway as a basis for going out and buying companies that already have a foothold in the European biosimilars space, whether European, Indian, Chinese, Korean or otherwise. Because the FDA is placing so much emphasis on analytical characterization and proof of similarity, brand manufacturers will be in a strong position to use their internal confidential business information about biomanufacturing processes in biosimilar subsidiaries to create new revenue streams.

But they are not the only stakeholders who will welcome the biosimilar pathway. Proponents of the need to cut healthcare costs will see in the guidances the first mapping of a detailed path by which equally effective but cheaper biotech medicines can reach American patients. And there is also some good news for patients: one of the FDA's stated goals is to create a bridge to the US market for biosimilars that are currently being sold in Europe, for which there is already substantial clinical trial information. Thus, new trials of patients for every US biosimilar may not be required.

The only actors who may be questioning whether this is good news or not are the biosimilar manufacturers themselves. And even here, non-US biosimilar companies can at least argue that they now have a route for US market access. The downside is that it appears unbearably onerous for all but the very few. 